\title{
Dirofilaria immitis (Leidy, 1856) no entorno de um caso felino: um estudo sobre sua transmissão
}

Dirofilaria immitis (Leidy, 1856) in the neighborhood of a feline case: a study about the transmission

Aline Serricella Branco ${ }^{1 *}$; Flavya Mendes-de-Almeida²; Maria Carolina Ferreira Faria ${ }^{3}$;

Letícia Mattos de Souza-Dantas²; Norma Vollmer Labarthe ${ }^{2}$

${ }^{1}$ Médica Veterinária, Universidade do Grande Rio - UNIGRANRIO

${ }^{2}$ Médica Veterinária, Universidade Federal Fluminense - UFF

${ }^{3}$ Médica Veterinária Autônoma, Universidade Federal Fluminense - UFF

Recebido em 28 de Agosto de 2008

Aceito em 4 de Setembro de 2009

\section{Resumo}

A espécie Dirofilaria immitis parasita principalmente canídeos, podendo infectar também gatos embora raramente (Felis catus Linnaeus, 1758). Seus hospedeiros intermediários podem ser diferentes espécies de culicídeos. Motivados por um caso autóctone de dirofilariose felina, encontrado na regiáo do Engenho Novo - RJ (S 22 90' e WO $43^{\circ} 27^{\prime}$ ), estudou-se: 1) a fauna culicídica local e; 2) a ocorrência de cáes portadores de microfilárias. Os estudos sobre a fauna culicídica foram conduzidos, de abril de 2003 a maio de 2004, realizando-se capturas peridomiciliares e intradomiciliares. Foram capturados 672 indivíduos (fêmeas) das espécies Aedes aegypti (Linnaeus) (561/672 - 83,5\%), Culex quinquefasciatus Say (96/672 - 14,3\%), Aedes (Oc.) scapularis (Rondani) (12/672 - 1,8\%) e Aedes albopictus (Skuse) (3/672-0,4\%). Apenas as espécies A. aegypti e C. quinquefasciatus foram capturadas em todos os meses do ano. Foram coletadas 235 amostras de sangue canino, durante a Campanha de Vacinação "Rio Sem Raiva", em outubro de 2003. Nenhuma amostra examinada continha microfilárias (técnicas de Knott e NAN), apesar de apenas 3,4\% dos cáes receberem quimioprofilaxia. A presença de culicídeos vetores, associada à possibilidade de mobilidade dos cáes, pode ter favorecido o aparecimento da infecção felina na região.

Palavras-chave: Dirofilariose felina, Vetores, Mosquitos.

\begin{abstract}
Dirofilaria immitis preferably infects canids and can, in lower frequency, be found in cats (Felis catus Linnaeus, 1758). The parasite may be transmitted by various Culicidae species. Motivated by an autochtone feline heartworm case diagnosed in the region of Engenho Novo - RJ ( $22^{\circ} 90^{\prime}$ e WO $\left.43^{\circ} 27^{\prime}\right)$, the following issues were surveyed: 1$)$ the local Culicidae fauna and; 2) the ocurrence of microfilaremic dogs. The mosquito study was conducted from April 2003 through May 2004, by peri-domiciliary and intra-domiciliary captures. A total of 672 mosquitoes (female) of the following species were caught: Aedes aegypti (Linnaeus) (561/672 - 83.5\%); Culex quinquefasciatus Say (96/672 - 14.3\%); Aedes (Oc.) scapularis (Rondani) (12/672 - 1.8\%) and Aedes albopictus (Skuse) (3/672 - 0.4\%). Only A. aegypti and C. quinquefasciatus specimens were captured year-round. A total of 235 canine blood samples were collected during the Campanha de Vacinação "Rio Sem Raiva" in October 2003. No microfilaria could be found in any of the examined samples (Knott's and NAN techniques), even though only 3.4\% of the dogs received chemoprophylaxis and the majority of them frequently traveled to endemic areas. The presence of vectors associated to a possible dog mobility, may have provided the right scenario for the feline D. immitis infection to happen in the studied area.
\end{abstract}

Keywords: Feline Heartworm, Vectors, Mosquitoes.

\footnotetext{
*Autor para correspondência: Aline Serricella Branco

Médica Veterinária, Universidade do Grande Rio - UNIGRANRIO,

Rua Passo da Pátria, 261, Duque de Caxias - RJ, Brasil

e-mail: aline-serricella@infolink.com.br
} 


\section{Introdução}

A espécie Dirofilaria immitis (Leidy, 1856) Raillet e Henry (1911) é o agente etiológico da infecção que pode acometer cães, gatos e seres humanos, dentre outras espécies animais (ATKINS et al., 2000; ATKINS et al., 2005; VITAL et al., 2006). Os canídeos são os hospedeiros definitivos aos quais esses helmintos são melhor adaptados (NELSON et al., 2005), entretanto, os relatos da infecção em felinos têm sido cada vez mais frequentes (ATKINS et al., 2000; ATWELL et al., 2001; CORNEGLIANI et al., 2003; ATKINS et al., 2005; LIU et al., 2005). A transmissão do parasito se dá por várias espécies de mosquitos da subfamília Culicinae, com diferentes taxas de eficiência (LUDLAN et al., 1970; SAUERMAN, 1980).

Indivíduos da espécie Felis catus não são hospedeiros definitivos de eleição de $D$. immitis, e a adaptação das duas espécies não é suficiente para permitir que os gatos sejam reservatórios de microfilárias, uma vez que a mortalidade entre eles, após a infecção, é alta, e a microfilaremia, quando ocorre, é transitória (DILLON, 2001; DILLON, 2003; NELSON et al., 2005; ATKINS; LISTER, 2006). Portanto, para que um gato seja infectado por $D$. immitis é necessário que, na mesma área, haja cáes portadores de microfilaremia e vetores competentes sejam atraídos e realizem repasto sanguíneos tanto em cães quanto em gatos (LABARTHE et al., 2002).

No Brasil, há poucos relatos de dirofilariose felina e, embora a infecção humana seja internacionalmente reconhecida como mais rara que a felina, aqui há mais registros de infecçôes humanas que felinas. É provável que, como o diagnóstico da infecção felina é difícil e o tratamento curativo impossível, os clínicos de Pequenos Animais não incluam essa possibilidade em seus diagnósticos diferenciais, contribuindo assim para que se tenha a impressão de que a infecção felina seja raríssima (LABARTHE et al., 2003).

Assim, com o intuito de descrever as condiçôes da regiâo onde um caso de infecção felina foi encontrado, conduziu-se o presente estudo buscando-se potenciais vetores e caninos portadores de microfilárias.

\section{Material e Métodos}

O estudo foi conduzido no bairro do Engenho Novo (Regiāo do Grande Meier), município do Rio de Janeiro, Estado do Rio de Janeiro (S $22^{\circ} 90^{\prime}$ e WO $43^{\circ} 27^{\prime}$ ), motivado por um caso autóctone de dirofilariose felina.

Uma gata sem raça definida, com 11 anos de idade, nasceu, foi criada e nunca saiu do bairro do Engenho Novo e teve morte, sem prenúncio de doença grave, em janeiro de 2003. Cerca de dois meses antes do óbito, o animal apresentou quadro de tosse e dispneia que, junto com o achado radiográfico de efusão pleural, foram interpretados, à época, como consequência de broncoaspiraçăo de óleo mineral que era administrado pela proprietária de forma rotineira, para a prevençáo de tricobezoar. A proprietária relatou que, na noite anterior ao óbito, o comportamento do animal era normal, inclusive brincando e que, pela manhã, encontrou o animal morto. À necropsia, um exemplar de nematoide foi encontrado no ventrículo direito e encaminhado ao Dr. Nicolau Maués da
Serra Freire (Universidade Estácio de Sá), que o identificou como D. immitis.

Foram realizadas capturas de culicídeos semanalmente, durante 12 meses consecutivos (maio de 2003 a abril de 2004), utilizando-se: (1) duas armadilhas luminosas do tipo CDC (Centers for Disease Control and Prevention, Atlanta, EUA ${ }^{1}$ com eliminação de $\mathrm{CO}_{2}$ (BATES, 1949); (2) pouso-homem, com auxílio de capturadores de Castro (BUXTON, 1980); e (3) aparato aspirador intradomiciliar (AC Insect Vac)2 (FORATTINI, 2002). Os mosquitos capturados foram identificados de acordo com as chaves taxonômicas de Lane (1953) e Consoli e Lourenço-de-Oliveira (1994).

Admitindo-se que a frequência de infecção canina por D. immitis, na regiáo do Grande Méier, estivesse entre 0,5 e $2 \%$ (LABARTHE et al., 1997), e que a população canina fosse de 5.000 animais (WHO, 1987), o número mínimo de amostras sanguíneas de cấes a ser examinado para garantir $80 \%$ de confiança foi 139 (Stacalc, EpiInfo 6, Centers for Disease Control and Prevention - CDC). Para tanto, durante a Campanha de Vacinaçáo "Rio Sem Raiva", da Prefeitura do Município do Rio de Janeiro (25 de outubro de 2003), após o consentimento livre e esclarecido de seus proprietários, obtiveram-se 235 amostras de sangue canino. As coletas foram realizadas nos postos de vacinação localizados a, no máximo, 800 metros do imóvel onde o caso de dirofilariose felina foi encontrado seguindo-se a dispersão máxima de $A$. aegypti conhecida (HONÓRIO et al., 2003) (Figura 1). Fichas identificadas foram preenchidas com informaçóes dos proprietários e dos cães.

As amostras sanguíneas foram examinadas quanto à presença de microfilárias pelo método de Knott (1939), modificado por Newton e Wright (1956) e NAN (ALMOSNY et al., 1991), no máximo três dias após a coleta. A técnica de NAN é semelhante à de Knott, substituindo a solução de formol por água destilada e solução de piperazina a $10 \%$, sem corantes. Sua vantagem é permitir a diferenciação específica das microfilárias.

O cálculo das frequências mensais dos mosquitos capturados para sua distribuição mensal foi realizado pela média de Williams (WILLIAMS, 1937; HADDOW, 1954). As diferenças entre frequência de capturas de culicídeos, usando-se diferentes métodos, foi avaliada pelo teste do Qui-quadrado e Fisher Exato (SAMPAIO, 2002), em tabela de contingência $2 \times 2$, com nível de significância igual a $5 \%$.

\section{Resultados}

Um total de 672 fêmeas de quatro espécies diferentes de culicídeos foi capturado e observou-se diferença entre o número total de mosquitos coletados por espécie $\left(\chi^{2}=1113,15 ; \mathrm{p}=0,000001\right)$ As quatro espécies foram Aedes aegypti (Linnaeus) (83,5\%), Culex quinquefasciatus Say (14,3\%), Aedes (Oc.) scapularis (Rondani) (1,8\%) e Aedes albopictus (Skuse) (0,4\%) (Tabela 1).

Houve diferença entre os métodos de coleta utilizados na captura tanto de $A$. aegypti $\left(\chi^{2}=364,61 ; \mathrm{p}=0,000001\right)$ quanto de $C$. quinquefasciatus $\left(\chi^{2}=63 ; \mathrm{p}=0,000001\right)$, mostrando que a

\footnotetext{
${ }^{1}$ American Biophysics, Jamestown, RI, EUA

${ }^{2}$ Bioquip Products, Califórnia, EUA
} 


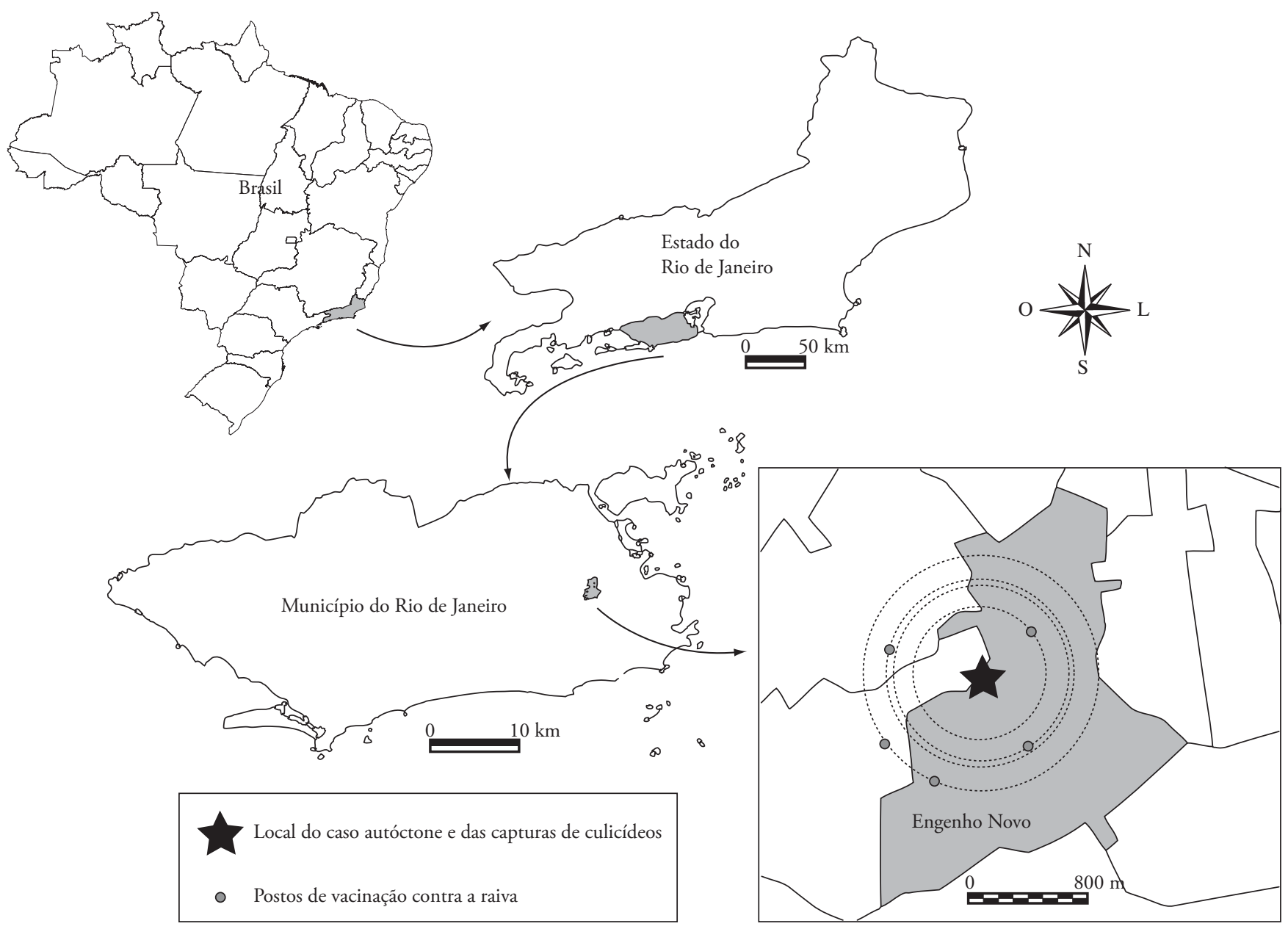

Figura 1. Mapas do Basil, do estado do Rio de Janeiro e da cidade do Rio de Janeiro, com destaque para o bairro do Engenho Novo

espécie $A$. aegypti foi capturada em maior número no ambiente intradomiciliar $\left(\chi^{2}=273,41 ; \mathrm{p}=0,000001\right)$ e C. quinquefasciatus, no ambiente peridomiciliar $\left(\chi^{2}=46,34 ; \mathrm{p}=0,000001\right)$ (Tabela 1 ) A espécie Aedes (Oc.) scapularis só foi encontrada no ambiente peridomiciliar e com maior frequência por pouso-homem $\left(\chi^{2}=13,50 ; \mathrm{p}=0,002\right)$. Não houve diferença entre os métodos de captura em relação à espécie $A$. albopictus. Apenas as espécies $A$. aegypti e $C$. quinquefasciatus puderam ser capturadas durante todo o ano. A ocorrência das espécies Aedes (Oc.) scapularis e A. albopictus foi baixa e intermitente ao longo do período (Figura 2).

Nenhuma microfilária foi encontrada nas 235 amostras de sangue canino examinadas, apesar de apenas oito $(3,4 \%)$ cáes receberem quimioprofilaxia.

Segundo informaçóes dos proprietários, a maioria dos cães examinados era mantida dentro de casa $(166 / 235$ - 71\%) e sem sair dos bairros onde residiam (197/235 - 84\%). Dentre os 38 cães que acompanhavam seus proprietários em viagens, $84 \%$ (32/38) frequentavam áreas onde há registros de transmissão de D. immitis.

A composição da população canina apresentou relação entre machos e fêmeas de 0,9:2, e a idade variou entre 1 e 17 anos, sendo a maioria dos animais com idade entre dois e seis anos.

\section{Discussão}

A única espécie de vetor já descrita, capturada no Estado do Rio de Janeiro como vetor primário para cáes Aedes $(O c$.) scapularis (LABARTHE et al., 1998), foi encontrada em baixa densidade. Por outro lado, C. quinquefasciatus, também capturada com frequência baixa, já foi apontada como vetor primário para gatos no Estado do Rio de Janeiro (LABARTHE et al., 1998), e na Itália (GENCHI et al., 1992). Além disso, sua taxa de infecçấo natural, no Brasil, varia entre 0,1 e 0,9\% (BRITO et al., 2001; LABARTHE et al., 1998) e, a artificial, entre 2,5 e 8,7\% (AHID et al., 2000). Apesar da densidade dos vetores influenciar na probabilidade de transmissão, não se pode afirmar que, quando em baixa densidade, aquela espécie não promova a transmissão (LUDLAM et al., 1970; PARKER, 1993). A espécie mais frequentemente capturada, $A$. aegypti, pode ter sido o vetor da infecção felina na regiáo do Grande Meier, uma vez que já foi apontada como vetor natural em outros países (VEZZANI et al., 2006) e como potencial vetor no Estado do Rio de Janeiro em estudo experimental (SERRÃO et al., 2001). Portanto, qualquer uma das três espécies capturadas em maior frequência pode ter realizado a transmissão, principalmente, porque mosquitos das três espécies já 
Tabela 1. Frequências absoluta e relativa de culicídeos capturados no bairro do Engenho Novo, Rio de Janeiro, usando-se diferentes métodos de captura.

\begin{tabular}{ccccc}
\hline \multirow{2}{*}{ Espécies capturadas } & \multicolumn{2}{c}{ Métodos de captura de culicídeos } \\
\cline { 2 - 3 } & \multicolumn{2}{c}{ Peridomiciliar } & & Intradomiciliar \\
\cline { 2 - 3 } Aedes aegypti & CDC & Pouso-homem & AID \\
\hline (Linnaeus) & $(8,20 \%)$ & $(21,21 \%)$ & & $(70,59 \%)$ \\
Culex quinquefasciatus & $66^{\mathrm{a}}$ & $8^{\mathrm{b}}$ & & $20^{\mathrm{c}}$ \\
Say & $(70,84 \%)$ & $(8,33 \%)$ & & $(20,83 \%)$ \\
Aedes (Oc.) scapularis & $1^{\mathrm{a}}$ & $11^{\mathrm{b}}$ & $0^{\mathrm{a}}$ \\
(Rondani) & $(8,33 \%)$ & $(91,7 \%)$ & $(-)$ \\
Aedes albopictus & $0^{\mathrm{a}}$ & $3^{\mathrm{a}}$ & $0^{\mathrm{a}}$ \\
(Skuse) & $(-)$ & $(100 \%)$ & $(-)$ \\
\hline
\end{tabular}

Letras diferentes nas linhas correspondem à diferença significativa ao nível de 5\% CDC - Centers for Disease Control and Prevention, Atlanta, EUA AID - aspirador intradomiciliar

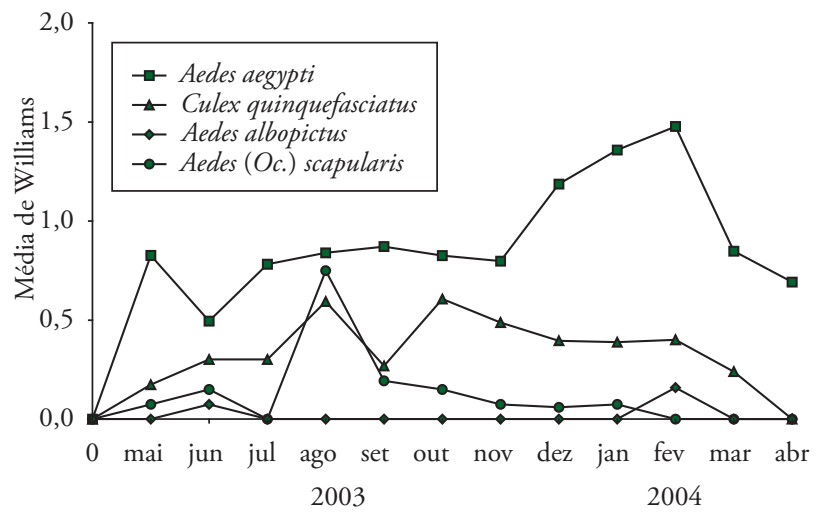

Figura 2. Frequências mensais de captura das espécies de mosquitos realizado pela Média de Williams, no Engenho Novo, Rio de Janeiro - Brasil, no período de maio de 2003 e abril de 2004.

foram capturados no Estado do Rio de Janeiro, contendo sangue canino e felino (GOMES, 2007).

Importante notar que a espécie Aedes (Oc.) scapularis só foi encontrada no peridomicílio, sugerindo que animais mantidos exclusivamente dentro de casa não seriam infectados por eles. Já as outras duas espécies foram capturadas tanto no domicílio quanto no peridomicílio, logo os animais poderiam ser infectados em qualquer um dos ambientes. Além disso, A. aegypti e C. quinquefasciatus poderiam transmitir o parasito a qualquer momento em que houvesse a presença de cães microfilarêmicos, uma vez que foram capturados durante todo o ano.

O fato de nenhum cáo examinado, em outubro de 2003, ser portador de microfilaremia, sugere que a frequência da infecção canina na região seja baixa. Entretanto, como 16\% deles acompanhavam seus proprietários em viagens para regióes endêmicas e náo faziam uso de medicação preventiva, é possível que casos de dirofilariose canina sejam, esporadicamente, introduzidos na região (GUERRERO et al., 1992; LABARTHE et al., 1997). Portanto, em um estudo de frequência na área, a probabilidade de encontrar um desses cáes durante uma campanha de vacinação é pequena.

Como infecçóes por $D$. immitis não são frequentes na região do Grande Meier, tanto Médicos Veterinários quanto proprietários deixam a populaçáo animal sem prevenção, concentrando grande quantidade de animais susceptíveis. Muitos desses hospedeiros susceptíveis circulam livremente entre áreas enzoóticas e indenes, garantindo a possibilidade de introdução do parasito na região estudada, onde há mosquitos de espécies vetoras (GUERRERO et al., 1992; LABARTHE et al., 1997). Os mosquitos locais, em baixa densidade populacional, são atraídos tanto por cães quanto por gatos (GOMES, 2007), o que demonstra que a ocorrência de dirofilariose felina na região, provavelmente casual, é possível. Entretanto, portadores de microfilaremia náo foram encontrados, o que pode parecer bizarro. Considerando-se que: 1) a gata que originou o estudo apresentou sintomatologia compatível com a infecção em novembro de 2002; 2) em gatos, os parasitos levam aproximadamente quatro meses para chegar às artérias pulmonares e ventrículo direito (DILLON, 2001; DILLON, 2003); 3) a sintomatologia felina ocorre com maior frequência e gravidade quando os parasitos chegam ao hábitat (sintomatologia precoce) ou quando morrem (TESKE, 1971; DILLON, 1984; DILLON, 1996; DILLON, 2001; CALVERT et al., 2003; DILLON, 2003; NELSON et al., 2005; ATKINS; LISTER, 2006) e; 4) a expectativa de vida dos parasitos, em gatos, é de aproximadamente dois anos (MCCALL et al., 1992; DILLON, 2001; DILLON, 2003; BAY, 2004; ATKINS; LISTER, 2006), pode-se admitir que a infecção da gata tenha ocorrido entre junho - julho de 2002 (caso a sintomatologia tenha sido precoce), ou no veráo de 2000/2001 (caso a sintomatologia tenha sido pela morte dos parasitos adultos). Portanto, é admissível que, passado tanto tempo, o cão ou cáes portadores de microfilaremia que infectaram os vetores já não estivessem mais na região, impossibilitando encontrar o animal reservatório.

Esses fatos sugerem que, em infecçôes por espécies de parasitos que cursam com período pré-patente longo e ciclo biológico complexo como $D$. immitis, não se deve negligenciar a vigilância epidemiológica e nem o uso de todo o arsenal preventivo disponível.

\section{Agradecimentos}

À Rosele Ribeiro e Celina Ribeiro (in memorian), por abrirem as portas de sua casa. À Maria Inês Dória Rossi, pela ajuda com o gráfico. À Heloisa Diniz, pelos mapas. À Núbia Karla de Almeida, pela estatística. A Mauro Salles Tupinambá, por todo apoio à realização deste trabalho. Ao Dr. Nicolau Maués da Serra Freire, pela identificação do exemplar de D. immitis. A todos os Médicos Veterinários que contribuíram para a realizaçáo deste estudo.

\section{Referências}

AHID, S. M. M. et al. Vector competence of Culex quinquefasciatus Say from different regions of Brazil to Dirofilaria immitis. Memórias do Instituto Oswaldo Cruz, v. 95, n. 6, p. 769-775, 2000.

ATKINS, C. E. et al. Heartworm infections in cats: 50 cases (1985-1997). Journal of the American Veterinary Medical Association, v. 217, n. 3, p. 355-358, 2000. 
ATKINS, C. E.; MORESCO, A.; LISTER, A. Prevalence of naturally occuring Dirofilaria immitis infections among non domestic cats housed in an area in wich heartworms are endemic. Journal of the American Veterinary Medical Association, v. 227, n. 1, p. 139-143, 2005.

ATKINS, C. E.; LISTER, A. L. Heartworm disease. In: AUGUST, J. R. Consultations in feline internal medicine. Missouri: Elsevier Saunders, 2006. 771 p. (v. 5).

BATES, M. The natural history of mosquitoes. New York: The Macmillan Company, 1949.

BAY, J. D. Dirofilariose. In: LAPPIN, M. R. Segredos em medicina interna de felinos. Porto Alegre: Atmed, 2004. 475 p.

BRITO, A. N. et al. Prevalência da filariose canina causada por Dirofilaria immitis e Dipetalonema reconditum em Maceió, Alagoas, Brasil. Cadernos de Saúde Pública, v. 17, n. 6, p. 1497-1504, 2001.

BUXTON, B. A.; MULLEN, G. R. Field isolation of Dirofilaria from mosquitoes in Alabama. Journal of Parasitology, v. 66, n. 1, p. 140-144, 1980 .

CALVERT, C. A.; RAWLINGS, C. A.; MCCALL, J. W. Dirofilariose. In: BIRCHARD, S. J.; SCHERDING, R. G. Manual Saunders: clínica de pequenos animais. São Paulo: Editora Roca, 2003. 1783 p.

CENTERS FOR DISEASE CONTROL AND PREVENTION - CDC Epi Info. 2002. Disponível em: <http://www.cdc.gov/epiinfo/downloads. htm>. Acesso em: Janeiro 2003.

CONSOLI, R. A. G. B.; LOURENÇO-DE-OLIVEIRA, R. Principais mosquitos de importância sanitária no Brasil. Rio de Janeiro: Fiocruz, 1994. 228 p.

CORNEGLIANI, L. et al. Two cases of cutaneous nodular dirofilariasis in the cat. Journal of Small Animal Practice, v. 44, n. 7, p. 316-318, 2003.

DILLON, R. Feline Dirofilariasis. Veterinary clinics of North America: small animal practice, v. 14, n. 6, p. 1185-1199, 1984.

DILLON, R. et al. Feline heartworm disease. Feline Practice, v. 24, n. 6, p. 12-16, 1996. (parte 1).

DILLON, R. Heartworm disease. In: MILLER, M. S.; TILLEY, L. P. Manual of canine and feline cardiology. 3 ed. Philadelphia: WB Saunders Co., 2001. 533 p.

DILLON, R. Feline heartworm disease: assessing the danger for owners. DVM Best Practices, p. 23-26, 2003. Disponível em: <http:// veterinarynews.dvm360.com/dvm/Parasitology+Center/Feline-heartwormdisease-Assessing-the-danger-for-/ArticleLong/Article/detail/59871>

FORATTINI, O. P. Culicidologia Médica. São Paulo: EDUSP, 2002.

GOMES, L. A. M. et al. Attraction of mosquitoes to domestic cats in a heartworm enzootic region. Journal of Feline Medicine and Surgery, v. 9, n. 4, p. 309-312, 2007.

HADDOW, A. J. Studies on the biting-habits of African mosquitoes: an appraisal of methods employed with special reference to the twentyfour-hour catch. Bulletin of Entomological Research, v. 45, p. 199-242, 1954.

HONÓRIO, N. A. et al. Dispersal of Aedes aegypti and Aedes albopictus (Diptera: Culicidae) in an urban endemic dengue area in the State of Rio de Janeiro, Brazil. Memórias do Instituto Oswaldo Cruz, v. 98, n. 2, p. 191-198, 2003.
LABARTHE, N. V. et al. Description of the occurrence of Canine Dirofilariasis in the State of Rio de Janeiro, Brazil. Memórias do Instituto Oswaldo Cruz, v. 92, n. 1, p. 47-51, 1997.

LABARTHE, N. V.; ALVES, L. C.; SERRÃO, M. L. Dirofilariose em pequenos animais domésticos e como zoonose. In: ALMOSNY, N. R. P. Hemoparasitose em pequenos animais domésticos e como zoonoses. Rio de Janeiro: L. F. Livros de Veterinária Ltda, 2002. 135 p.

LABARTHE, N.; ALVES, L. C.; SOUZA-DANTAS, L. M. Dirofilariose. In: SOUZA, H. J. M. (Ed.) Coletânea em medicina e cirurgia felina. Rio de Janeiro: L.F. Livros de Veterinária Ltda, 2003. 475 p.

LABARTHE, N.V.; SERRÁO, M.L.; MELO, Y.F.; OLIVEIRA, S.J.; LOURENÇO-DE-OLIVEIRA, R. Potential Vectors of Dirofilaria immitis (Leidy, 1856) in Itacoatiara, Oceanic Region of Niterói Municipality, State of Rio Janeiro, Brazil. Memórias do Instituto Oswaldo Cruz, v. 93, n. 4, p. 425-432, 1998.

LANE, J. Neotropical culicida. São Paulo: Universidade de São Paulo, 1953.

LIU, J. et al. Serological and molecular survey of Dirofilaria immitis infection in stray cats in Gyunggi province, South Korea. Veterinary Parasitology, v. 130, n. 1/2, p. 125-9, 2005.

LUDLAM, K. W.; JACHOWSKI, L. A.; OTTO, G. F. Potential vectors of Dirofilaria immitis. Journal of the American Veterinary Medical Association, v. 157, n. 10, p. 1354-1359, 1970.

NELSON, C. T. et al. Guidelines for the diagnosis, prevention and management of heartworm (Dirofilaria immitis) infection in cats. Veterinary Parasitology, v. 133, n. 2/3, p. 267-75, 2005.

NEWTON, W. L.; WRIGHT, W. H. The occurrence of a dog filariid other than Dirofilaria immitis in the United States. Journal of Parasitology, v. 42, n. 3, p. 246-258, 1956

PARKER, B. M. Variation in mosquito (Diptera: Culicidae) relative abundance and Dirofilaria immitis (Nematoda: Filaroidea) vector potential in coastal North Carolina. Journal of Medical Entomology, v. 30, n. 2, p. 436-442, 1993.

SAMPAIO, I. B. M. Estatística aplicada à experimentação animal. 2 ed. Belo Horizonte: FEPMVZ, 2002. 265 p.

SERRÃO, M. L.; LABARTHE, N.; LOURENÇO de OLIVEIRA, R. Vectorial competence of Aedes aegypti (Linnaeus 1762) Rio de Janeiro Strain, to Dirofilaria immitis (Leidy 1856). Memórias do Instituto Oswaldo Cruz, v. 96, n. 5, p. 593-598, 2001.

TESKE, R. H. Dirofilariasis in a cat. Journal of the American Veterinary Medical Association, v. 159, n. 8, p. 891, 1971.

VEZZANI, D.; EIRAS, D. F.; WISNIVESKY, C. Dirofilariasis in Argentina: Historical review and first report of Dirofilaria immitis in a natural mosquito population. Veterinary Parasitology, v. 136, n. 3/4, p. 259-273, 2006.

VITAL, R. J.; MATTOS, L. A.; MEIRELLES, G. S. P. Human pulmonary dirofilariasis: atypical presentation of a rare disease. Revista da Sociedade Brasileira de Medicina Tropical, v. 39, n. 1, p. 94-95, 2006.

WORLD HEALTH ORGANIZATION - WHO. Guidelines for dog population management. Geneva: WHO, 1987.

WILLIAMS, C. B. The use of logarithms in the interpretation of certain entomological problems. Annals of Applied Biology, v. 24, n. 2 , p. 404-414, 1937. 